\title{
EL PLURAL EN LOS SUSTANTIVOS TERMINADOS EN VOCAL EN EL CANTON DE CURRIDABAT: ESTUDIO CUANTITATIVO
}

\author{
Annette Calvo Shadid
}

\begin{abstract}
This study presents a quantitative distribution of plural variants for vowel-ending nouns observed in Curridabat county, province of San José. The purpose of the research is to determine the relationship, first, between the linguistic context which determines the word chosen and the plural variants used and, second, between these variants and the social variables of age, sex and level of education of the speakers.
\end{abstract}

\section{Introducción}

\subsection{Estudios previos}

En español, existe una gran cantidad de estudios realizados acerca de la marcación de la categoría plural de los sustantivos. Sin embargo, en ninguno de ellos se ha estudiado la variación existente en el uso de las diferentes formas del plural. Lo que se ha hecho, ha sido proponer reglas de distribución de las variantes con base en diversos enfoques teóricos.

En este sentido, se pueden clasificar las distintas investigaciones en tres grupos: tradicionales (prescriptivas), estructurales (descriptivas) y generativo transformacionales.

Dentro de los trabajos prescriptivos se ha destacado el de la Real Academia Española (1920: 18-20). En él se proponen reglas para la formación del plural de acuerdo con dos variantes:

es: con sustantivos terminados en consonante y en vocal acentuada que no sea $e$, con algunas excepciones como papás, maravedís, mamás, chacós, rondós;

-s: con sustantivos terminados en vocal inacentuada y en é acentuada (é). Además, reconoce que hay sustantivos "que no consienten alteración alguna en el plural", (Real Academia 1920: 18-9) como son los que presentan más de una sílaba y terminan en -s: Carlos, crisis, dosis, éxtasis, etc. ${ }^{1}$.

Los autores costarricenses de gramáticas como Gagini (1920:57), Marín (s.d.: 23), Agüero (1962-147-8), Quesada (1929:147-50), González (1969:37), Zamora Elizondo (194190-3), siguen el criterio de la Real Academia (1920) para la formación del plural.

Dentro de los estudios de enfoque estructuralista, se encuentra Saporta (1965:218-24), quien formula la siguiente regla para la distribución de tres variantes de plural:

-s: es el plural de los sustantivos terminados en vocal inacentuada y en vocal e acentuada (é);

$-\phi$ : es el plural de los sustantivos terminados en vocal inacentuada más $-s$;

es: es el plural de los sustantivos terminados en consonante y en vocal acentuada que no sea é.

Alcina y Blecua (1975:536-8) apuntan las mismas variantes del plural, pero para las palabras terminadas en vocal acentuada reconocen que hay alternancia entre -es y $-s$. 
Carratalá (1980:68-71) sigue los criterios de Saporta para la distribución de los tres variantes de plural. Además, presenta "excepciones" para los sustantivos terminados en vocal acentuada, por una tendencia "actual" a hacer algunos de sus plurales en $-\mathrm{s}^{2}$

Dentro de los estudios generativos transformacionales se encuentra a Foley (1967: 48693), quien propone la existencia de un solo formativo de plural para todos los sustantivos: -s. Para explicar las alternancias, supone que toda palabra terminada en consonante o en vocal tónica tiene una $e$ subyacente, la cual, por una regla de apócope se suprime en singular, pero permanece en plural.

Saltarelli (1970:89-96), en oposición a la teoría de la apócope de Foley, propone que, tal como existe una regla de prótesis en español ( ${ }^{*}$ sposa $\rightarrow$ e-sposa), también hay una regla de epéntesis, por la que se agrega, al hacer el plural, una - $e$ a la forma $-s$ que corresponde al plu$\mathrm{ral}^{3}$.

Por otro lado se han hecho numerosos estudios acerca de la formación del plural de los dialectos del castellano en los cuales hay elisión de /-s/ (lo cual no se da en el español de Costa Rica). El primero de ellos fue el de Navarro (1939) para el español andaluz peninsular, cuya elisión de /-s/ lo lleva a proponer que, debido a esto, se da una apertura de la vocal precedente. Ese rasgo permite establecer diferencias semánticas significativas al convertirse en el marcador del plural nominal: /diénte/ 'diente': /diéntę/ 'dientes'. De este modo se da un desdoblamiento vocálico y, de un sistema de cinco vocales se pasaría a uno de siete.

Hooper (1976:34-5), presenta el mismo argumento para el español de Andalucía, particularmente para Andalucía Oriental, cuyo plural se marca por aspiración y por la apertura de la vocal final. Esta diferencia de vocales abiertas y cerradas se describe como diferencias de tensión:

$$
\mathrm{V} \rightarrow>\text { [- tensa] / I+ plural] (h) \#\# }
$$

Encuentra que el proceso de reducción de la tensión vocálica ha llegado a morfologizarse, mientras que la motivación fonética de la vocal laxa (la elisión o aspiración de /-s/) todavía es opcional en la lengua.
Basada en Alonso et al (1950) muestra que, en el dialecto de Granada, la apertura de la vocal final, en plural, se extiende a toda la

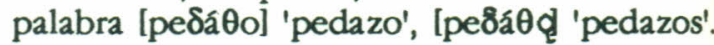
Considera este rasgo como marcador de una categoría morfológica.

Algunos estudios dialectológicos hispanoamericanos muestran el mismo fenómeno para los dialectos que eliden el fonema /-s/ final: Vázquez (1953) para Uruguay, Matluck (1961) para Puerto Rico, Honsa (1965) para Argentina, Haden y Matluck (1973) para Cuba, entre otros.

En otros trabajos posteriores también se ha demostrado que es imposible sostener el argumento del desdoblamiento vocálico al elidirse la /-s/ final en la formación del plural: Cedergren (1973) para Panamá, Terrel (1977a) para Cuba y Puerto Rico, Poplack (1980) para Puerto Rico, Alba (1982) para Santiago de los Caballeros, Hundley (1987) para Perú.

Otros estudios comprueban, por medio de los análisis acústicos y de percepción que la mencionada fonologización de las vocales es insostenible, por lo menos en lo que respecta a dialectos del español en América: Clegg (1967) para Cuba, Hammond (1976) para el español cubano de Miami, Alemán (1977) para Puerto Rico, y Cedergren (1978) para Panamá.

Para Terrel (1977b: 41) y· López Morales (1971, 1983:51), la regla de elisión de /-s/ desempeña un papel importante en la gramática, debido a que funciona como indicador de plural nominal y está determinada por factores morfológicos, aunque las condiciones de distintividad se relacionan con la redundancia de las estructuras externas: si la marca es redundante hay mayor elisión; esto es, la regla de elisión apunta a la simplificación de la redundancia en las estructuras externas.

\subsection{Propósito de la investigación}

El presente trabajo tiene como finalidad establecer una distribución cuantitativa de las variantes de plural en los sustantivos terminados en vocal, de acuerdo con dos hipótesis:

a. Se supone que existe relación entre el contexto lingüístico que determina la palabra y la variante de plural usada. 
b. Se supone que existe relación entre las variables de edad, sexo y nivel de instrucción de los hablantes y las variantes de plural usadas.

\section{Metodologia}

En este estudio se tomó en cuenta la variable lingüística de plural, la cual presenta diversas realizaciones o variantes distribuidas a lo largo de tres variables sociales. Las variantes lingüísticas observadas fueron: operación cero4, $-s-e s,-$ ses y otros.

Para el estudio morfológico de las alternancias se utilizó un cuestionario compuesto por 83 ítemes, los cuales presentaban diferentes terminaciones. Los encuestados educían las formas del plural a partir del singular que se les daba.

Para la recolección de los datos se tomó una muestra por cuota manteniendo las proporciones de sexo, nivel de instrucción y grupos de edades observadas en el cantón de Curridabat, provincia de San José de Costa Rica. En relación con la selección de las variables sociales se siguieron las pautas de López Morales (1983: 25-6).

La edad quedó delimitada de la siguiente manera: I generación, 25 a 35 años; II generación, 36 a 55 años; III generación, 56 años en adelante.

La variable nivel de instrucción se estratificó según tres niveles: primaria, secundaria y universitaria.

De acuerdo con los recursos, el tiempo y los objetivos del estudio, se tomó una muestra del $0.25 \%$ de la población, distribuida como se presenta en el Cuadro 1.

\section{CUADRO 1}

Curridabat: Distribución de la población según nivel de instrucción por grupos de edad y sexo 1984

\begin{tabular}{|c|c|c|c|c|c|c|c|}
\hline \multirow[t]{2}{*}{ Instrucción } & \multicolumn{3}{|c|}{$\mathrm{H}$} & \multicolumn{3}{|c|}{$M$} & \multirow[t]{2}{*}{ Total } \\
\hline & $23-35$ & $35-55$ & 55 y más & $25-35$ & $35-55$ & 56 y más & \\
\hline Primaria & 2 & 3 & 2 & 3 & 4 & 2 & \\
\hline Secundaria & 2 & 1 & 1 & 3 & 2 & 1 & 10 \\
\hline Universitaria & 2 & 2 & 0 & 2 & 1 & 0 & \\
\hline Total & 6 & 6 & 3 & 8 & 7 & 3 & \\
\hline
\end{tabular}

Además, se tomaron en cuenta las siguientes variantes de control (cfr. Lope Blanch 1977:9):

a. Como el estudio se limitó al cantón de Curridabat, se observó que los sujetos hubieran nacido alli o que residieran en el cantón desde los cinco años.

b. Que fueran hijos de hispanohablantes nacidos en el área metropolitana.

Para el procedimiento de la información se utilizó el paquete estadístico SPSS (Statistical Packet for Social Sciences).

\section{Distribución de las variantes lingüisticas:}

\subsection{Vocales}

\subsubsection{Vocales inacentuadas}

\section{CUADRO 2}

Distribución de las variantes de plural en palabras terminadas con vocal inacentuada (términos porcentuales)

\begin{tabular}{|c|c|c|c|c|c|c|c|}
\hline \multirow{2}{*}{$\begin{array}{l}\text { Vocales } \\
\text { inacentuadas }\end{array}$} & \multicolumn{6}{|c|}{ Variantes } & \multirow[b]{2}{*}{ Total } \\
\hline & op. $\phi$ & $-s$ & -es & -ses & otros & NS/NR & \\
\hline a & 4.5 & 95.5 & - & - & - & - & 100 \\
\hline e & 0 & 100 & - & - & - & - & 100 \\
\hline o & 9.1 & 89.9 & 1.0 & - & - & - & 100 \\
\hline i & 36.4 & 60.6 & 3.0 & - & - & - & 100 \\
\hline
\end{tabular}

En las palabras terminadas con vocal inacentuada, la tendencia general es agregar sufijo $-s$.

Sin embargo, para la vocal $-i$, se observa una variación interesante que tiende, en un porcentaje alto $(36,4 \%)$, a la marcación de la operación cero.

Esto podría deberse a que, regularmente, en castellano no se dan palabras que terminen con la vocal inacentuada -i. La que se utilizó en el cuestionario fue jersey [yérsi]; esta es de origen extranjero, y aparentemente tiene un uso restringido.

Con las otras vocales, se cumple casi en un $100 \%$ la regla expuesta por la mayor parte de los autores: las palabras terminadas en vocal inacentuada agregan el sufijo $-s$. 


\subsubsection{Vocales acentuadas}

\section{CUADRO 3}

Distribución de las variantes de plural en palabras terminadas con vocal acentuada (términos porcentuales)

\begin{tabular}{c|r|r|r|r|r|r|r}
\hline \multirow{2}{*}{$\begin{array}{l}\text { Vocales } \\
\text { acentuadas }\end{array}$} & \multicolumn{6}{|c|}{ Variantes } & \multirow{2}{*}{} \\
\cline { 2 - 7 } & oper. $\phi$ & -s & -es & -ses & otros & NS/NR & Total \\
\hline E & 9.1 & 84.8 & - & 6.1 & - & - & 100 \\
a & 20 & 40 & 15.2 & 23.6 & 1.2 & - & 100 \\
6 & 21.8 & 45.2 & 27.9 & 3.6 & 1.2 & 0.3 & 100 \\
f & 12.9 & 21.2 & 62.5 & 3.4 & - & - & 100 \\
f & 17 & 25.8 & 48.1 & 8.7 & - & 0.4 & 100
\end{tabular}

Los porcentajes que se presentan en el Cuadro 3 muestran cómo las vocales altas acentuadas $(-\boldsymbol{i},-\boldsymbol{i})$ presentan una tendencia mayor a hacer los plurales con el sufijo -es (62.5\% y $48.1 \%$, respectivamente).

En segundo lugar, estas vocales presentan el uso del exponente $-s(21.2 \%$ y $25.8 \%$, respectivamente).

Por el contrario, las vocales bajas acentuadas $(-\hat{a},-\hat{O})$ muestran preferencia por los plurales con $-s$ ( $40 \%$ y $45.2 \%$, respectivamente), luego por los plurales con -es $(15.2 \%$ y $27.9 \%$, respectivamente) y por medio de la operación cero ( $20 \%$ y $21.8 \%$, respectivamente), frente a las vocales altas $(-\hat{i},-\hat{i})$ las cuales muestran un porcentaje un tanto más bajo en el uso de este último exponente $(12.9 \%$ y $17 \%$ respectivamente).

Las palabras terminadas en -é se excluyen de estos grupos debido a que, por una ley fonética del español, estas no pueden tener la forma, por ejemplo, "cafées, sino sólo cafés, café o cafeses.

El uso del exponente -ses es bajo, en general, excepto en la vocal -á, la cual se tratará por aparte. De acuerdo con los porcentajes que presentan las otras vocales en esta casilla, no es posible establecer ninguna correlación fonológica.

En el cuestionario se incluyeron dos ítemes que terminan en la vocal acentuada $-\hat{a}$, los cuales se usan en el área estudiada prácticamente solo en plural, y estos ya se han generalizado de una forma; tales son tirrá-tirrases (topónimo del cantón de Curridabat) y cubá-cubases (un tipo de frijol).
Las plurales de estas palabras dieron el siguiente resultado:

CUADRO 3.1

Distribución de las variantes de plural en palabras terminadas con -a acentuada (Términos porcentuales)

\begin{tabular}{l|r|r|r|r|r|l}
\hline \multirow{2}{*}{ Vocal a } & \multicolumn{5}{|c|}{ Variantes } & \multirow{2}{*}{} \\
\cline { 2 - 6 } & op. $\phi$ & -s & es & ses & otros & Total \\
\hline \multirow{2}{*}{ tirá } & 16 & 33 & 3 & 48 & - & 100 \\
cubá & 6 & 36 & - & 58 & - & 100 \\
atarrá & 15 & 55 & 18 & 9 & 3 & 100 \\
agrá & 30 & 42 & 22 & 3 & 3 & 100
\end{tabular}

Como los plurales de las palabras tirrá y cubá están ya lexicalizados, afectan el resultado de la variante -ses en el contexto indicado, pues en las otras palabras (atarrá y agrá) aparece el uso de -ses solamente nueve veces, en el primer caso, y tres veces en el segundo.

Las variantes otros no mostró resultados significativos; sin embargo; se observó un caso interesante en cinco palabras: dos terminadas en -á: atarranes y agrán, y tres en $-\sigma$ : burones, bongones, rondones.

Esto puede interpretarse de varias formas: 0 -nes es otro exponente de plural para los sustantivos, o es el resultado de una doble pluralización de $-\boldsymbol{n}$, que es el formativo de plural de verbos (como en duermen, bacen etc.), y de -es, que es formativo de plural de sustantivos.

Por otro lado, la variante - $\boldsymbol{n}$ en agrán podría corroborar que el plural se hizo, en ese caso, acudiendo al formativo de plural $\boldsymbol{n} \boldsymbol{n}$ de los verbos.

El Dr. Sánchez Corrales (comunicación personal) sugirió otra explicación para este fenómeno, la cual se relaciona con el proceso de velarización y elisión de $-\boldsymbol{n}$ en posición final.

Se ha demostrado que $/ \mathrm{n} /$ final, en el espanol del Caribe, sufre un proceso de debilitamiento que se manifiesta o en la velarización $[\eta]$ o en la elisión $[\phi]^{5}$.

Las palabras cuyo plural fue hecho en $-n$ o -nes terminan en vocal acentuada. Según lo manifestado en el párrafo anterior, los informantes pudieron haber escuchado estos ítemes con - $\boldsymbol{n}$ final que hubiera sido debilitada: [agrán], [agŕa $\phi$ ]; [buró con palabras como ratón o canción, que sí la tiene fonéticamente debilitada. 


\section{CUADRO 4}

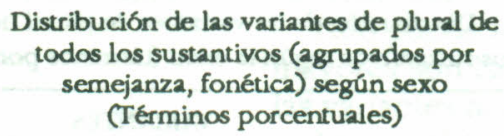

\begin{tabular}{|c|c|c|c|c|c|c|c|c|c|c|c|c|}
\hline \multirow[b]{3}{*}{ Entornos } & \multicolumn{10}{|c|}{ VARIANTES } & & \\
\hline & \multicolumn{2}{|c|}{ op. $\phi$} & \multicolumn{2}{|c|}{$-s$} & \multicolumn{2}{|c|}{ es } & \multicolumn{2}{|c|}{-ses } & \multicolumn{2}{|c|}{ otros } & \multicolumn{2}{|c|}{ NS/NR } \\
\hline & $\mathbf{M}$ & $\mathrm{F}$ & $\mathbf{M}$ & $\mathbf{F}$ & $\mathbf{M}$ & F & $\mathbf{M}$ & $\mathbf{F}$ & $\mathbf{M}$ & $\mathbf{F}$ & M & F \\
\hline $\mathrm{V}$ inacent. & 8.6 & 12 & 89.4 & 87.3 & 2 & 0.7 & - & - & - & - & - & - \\
\hline V bajas acen. & 15 & 20.8 & 55.3 & 52.2 & 23.3 & 13.3 & 6 & 12 & 0.4 & 1.4 & - & 0.3 \\
\hline $\mathrm{V}$ altas acen. & 14.2 & 19.4 & 26.7 & 25 & 57.5 & 40.3 & 1.7 & 14.6 & - & - & - & 0.7 \\
\hline
\end{tabular}

\section{Distribución de las variables sociales}

\subsection{Variable sexo}

En general, en este punto se presentan algunas diferencias que pueden considerarse representativas entre las variantes masculino y femenino; por lo tanto, podría pensarse que esta variable influye en la formación del plural de algunos de estos sustantivos.

En las mujeres, se observa una tendencia mucho mayor a formar el plural de las vocales acentuadas con la variante -ses.

Así mismo, en las mujeres se presentan porcentajes más altos en el uso de la operación cero de plural en los tres entornos.

Por otro lado, en los hombres se presenta una tendencia mayor a usar la variante, -es, sobre todo en las vocales acentuadas.

A partir de este análisis, se puede concluir que las mujeres, en la muestra observada, tienden a hacer un mayor uso de las variantes innovadoras, o más bien, menos normativas del plural, como -ses y la operación cero.

Esto corrobora lo que ya se ha observado en otros estudios sobre el español de América: el hecho de que es la mujer la que propicia el cambio lingüístico en una proporción mayor que el hombre. En este sentido, Perissinotto (1972) demuestra que la mujer va adelante en el proceso de asibilación de las vibrantes en la ciudad de México.

En relación con el yeísmo porteño, Wolf y Jiménez (1977) demuestran cuantitativamente una diferencia considerable debida al sexo: las mujeres utilizan la variante ensordecida en un porcentaje mucho mayor que los hombres.
De acuerdo con los resultados, las variantes de plural preferidas por la variable sexo son las siguientes:

\section{CUADRO 4.1}

Preferencia por las variantes de plural según el sexo (Términos porcentuales)

\begin{tabular}{l|r|r|r|r|r|r|r}
\hline \multirow{2}{*}{ Sexo } & \multicolumn{6}{|c|}{ Variantes } & \multirow{2}{*}{ Total } \\
\cline { 2 - 7 } & op. $\phi$ & -s & -es & -ses & otros & NS/NR & \\
\hline M & 12.6 & 57.1 & 27.6 & 2.5 & 0.2 & - & 100 \\
F & 17.5 & 54.8 & 18 & 8.9 & 0.5 & 0.3 & 100
\end{tabular}

El cuadro anterior corrobora que las diferencias más importantes se observan en el uso mayor de la operación cero y de la variante -ses en el sexo femenino, y un mayor uso de las variantes más normalizadas de plural ( $-s$ y es) en el sexo masculino.

\subsection{Variable edad}

Esta variable muestra diferencias interesantes en las tres generaciones:

En primer lugar, la diferencia más importante se da en el uso de la operación cero: en todos los entornos, la III generación presenta una tendencia mayor de uso que los otros dos grupos (sobre todo en las vocales acentuadas). Además, la II generación presenta porcentajes bastante mayores que la I en las vocales inacentuadas y en las altas acentuadas.

Otra situación interesante se da con la variante -ses. En los dos entornos en que se 


\section{CUADRO 5}

Distribución de las variantes de plural de todos los sustantivos según la edad (términos porcentuales)

\begin{tabular}{|c|c|c|c|c|c|c|c|c|c|c|c|c|c|c|c|c|c|c|}
\hline \multirow[b]{3}{*}{ Entornos } & \multicolumn{18}{|c|}{ VARIANTES } \\
\hline & \multicolumn{3}{|c|}{ op. $\phi$} & \multicolumn{3}{|c|}{$-s$} & \multicolumn{3}{|c|}{ es } & \multicolumn{3}{|c|}{-ses } & \multicolumn{3}{|c|}{ otros } & \multicolumn{3}{|c|}{ NS/NR } \\
\hline & I & II & III & I & III & III & I & II & III & $\mathrm{I}$ & III & III & $\mathrm{I}$ & II & III & I & III & III \\
\hline in & 7.7 & 11.2 & 14.3 & 90.1 & 87.8 & 85.7 & 2.2 & 1 & - & - & - & - & - & - & - & - & - & - \\
\hline $\mathrm{V}$ bajas acen. & 14.2 & 14.3 & 35.8 & 67.4 & 50.5 & 30.8 & 13.4 & 19 & 25 & 4.6 & 14.7 & 6.7 & 0.4 & 1.5 & 1.7 & - & - & - \\
\hline $\mathrm{V}$ altas acen. & 7.7 & 17.9 & 35.4 & 39.4 & 20.5 & 8.3 & 48.1 & 47.3 & 50 & 3.8 & 14.3 & 6.3 & - & - & - & 1.0 & - & - \\
\hline
\end{tabular}

presenta, el porcentaje mayor se da en la II generación. En segundo lugar se da en la III generación y, en tercero, con un porcentaje bajo, en la I generación.

En el caso de la variante - $s$, la primera generación presenta los porcentajes más altos, y la III generación los más bajos.

En la variante -es se presentan diferencias poco significativas y no sistemáticas.

\section{CUADRO 5.1}

Preferencia por las variantes de plural según la edad (términos porcentuales)

\begin{tabular}{c|r|r|r|r|r|r|c}
\hline \multirow{2}{*}{ Generación Variantes } & \\
\cline { 2 - 8 } & op. $\phi$ & -s & -es & -ses & otros & NS/NR & Total \\
\hline \multirow{2}{*}{ I } & 10 & 65.7 & 21.4 & 2.7 & 0.2 & - & 100 \\
II & 14.5 & 52.9 & 22.4 & 9.7 & 0.5 & - & 100 \\
III & 28.4 & 41.5 & 25 & 4.2 & 0.6 & 0.3 & 100
\end{tabular}

En general, las mayores diferencias se observan en la tercera generación, en la que se da una marcada preferencia por la operación cero, en detrimento de la variante $-s$, y en la segunda generación, cuya preferencia por la variante -ses es mayor que en las otras dos generaciones.

\subsection{Variable nivel de instrucción:}

Esta variable también presenta algunas diferencias interesantes a lo largo de sus tres niveles:

En el nivel de secundaria, se observa una tendencia mayor que en los niveles de primaria y universitaria a usar la operación cero en todos los entornos, y también un uso significativamente menor de la variante es.

La variante -ses en las vocales acentuadas, muestra una marcada preferencia en el nivel de primaria, con respecto a los otros dos niveles de instrucción.

\section{CUADRO 6}

Distribución de las variantes de plural de todos los sustantivos según el nivel de instrucción (términos porcentuales)

\begin{tabular}{|c|c|c|c|c|c|c|c|c|c|c|c|c|c|c|c|c|c|c|}
\hline \multirow[b]{3}{*}{ Entornos } & \multicolumn{18}{|c|}{ VARIANTES } \\
\hline & \multicolumn{3}{|c|}{ op. $\phi$} & \multicolumn{3}{|c|}{$-s$} & \multicolumn{3}{|c|}{-es } & \multicolumn{3}{|c|}{$-\operatorname{ses}$} & \multicolumn{3}{|c|}{ otros } & \multicolumn{3}{|c|}{ NS/NR } \\
\hline & $\mathbf{P}$ & S & $\mathrm{U}$ & $\mathbf{P}$ & $S$ & $\mathrm{U}$ & $\mathbf{P}$ & $S$ & $\mathrm{U}$ & $\mathbf{P}$ & $\mathrm{S}$ & $\mathbf{U}$ & $\mathbf{P}$ & $\mathrm{S}$ & $\mathbf{U}$ & $\mathbf{P}$ & $\mathrm{S}$ & $\mathrm{U}$ \\
\hline $\mathrm{V}$ inacent. & 8.6 & 15.9 & 7.9 & 90.5 & 84.1 & 88.9 & 0.9 & - & 3.2 & - & - & - & - & - & - & - & & - \\
\hline $\mathrm{V}$ bajas acen & 10.7 & 28.9 & 20 & 51 & 63.3 & 48.3 & 24.6 & 2.8 & 21.6 & 12 & 5 & 8.9 & 1.7 & - & 0.5 & - & & 0.5 \\
\hline $\mathrm{V}$ altas acen & 13.3 & 29.2 & 11.1 & 19.2 & 47.2 & 15.3 & 53.3 & 23.6 & 63.9 & 14.2 & - & 8.3 & - & - & - & - & & 1.4 \\
\hline
\end{tabular}


El siguiente cuadro las variantes de plural preferidas de acuerdo con el nivel de instrucción:

\section{CUADRO 6.1}

Preferencia por las variantes de plural según el nivel de instrucción (términos porcentuales)

\begin{tabular}{l|r|r|r|r|r|r|r}
\hline \multirow{2}{*}{$\begin{array}{l}\text { Nivel de } \\
\text { instrucción }\end{array}$} & \multicolumn{6}{|c|}{ Variantes } & \\
\cline { 2 - 8 } & op. $\phi$ & -s & -es & -ses & otros & NS/NR & Total \\
\hline P & 10.6 & 53.6 & 26.3 & 8.7 & 0.5 & - & 100 \\
S & 24.7 & 64.9 & 8.8 & 1.7 & - & - & 100 \\
U & 13 & 50.1 & 29.6 & 5.7 & 0.1 & 0.6 & 100
\end{tabular}

En este cuadro se corroboran las diferencias observadas arriba.

\section{Conclusiones:}

Por medio de los resultados de la investigación realizada, se llegó a las siguientes conclusiones:

Existe una relación estrecha entre el contexto que determina la palabra y el formativo de plural usado.

En relación con esto, se da una tendencia general a formar los plurales de los sustantivos terminados en vocal inacentuada con la variante $-s$.

Las palabras terminadas en vocal acentuada presentan una mayor variación en la formación del plural, la cual parece estar relacionada con la altura de estas vocales.

En las vocales altas acentuadas $(-i,-i)$ se prefiere la variante $-e s$, y en las bajas $(-\hat{a},-\hat{O})$, se observa un uso mayor del formativo $-s$.

Las variables sociales no permiten establecer una relación directa entre ellas y las variantes de plural. Sin embargo, al igual que en otros estudios cuantitativos hechos en el español de América, se observó que las mujeres utilizan más que los hombres las variantes menos normativas (la operación cero y -ses) en los entornos en los que no se espera que ocurra.

La variable edad dio resultados un tanto diferentes, pues la tercera generación tiene una marcada preferencia por la operación cero, pero es la segunda la que la tiene por la variante - ses.

Esto puede indicar que las dos generaciones mayores son las que usan más frecuentemente las variantes menos normativas.

La variable nivel de instrucción presentó resultados similares a los anteriores: en el nivel de secundaria se observó un uso mayor de la operación cero, y en el de primaria, se da preferencia por la variante -ses.

En resumen, las variables sociales que muestran preferencia por las variantes menos normativas son el sexo femenino, la II y III generaciones, y los niveles de primaria y secundaria.

Esto indica que los resultados obtenidos no permiten concluir qué variables sociales están facilitando un cambio lingüístico.

\section{Notas}

1. Entre otros estudios generales de tipo tradicional que siguen lo prescrito por la Real Academia, se pueden citar: Bello (1847), Lenz (1925), Garcia de Diego (1951), Henríquez Ureña (1949), Pérez Rioja (1954), Real Academia Española (1973), Martínez de Sousa (1974), De la Peña (1985).

2. Otros estudios de tipo estructuralista que se pueden citar: Stockwell et al (1965), Lorenzo (1971), Sánchez (1988).

3. Otros estudios generativos transformacionales que se pueden citar: Hadlich (1973), Harris (1985), Cepeda (1980).

4. La operación cero (u operación de identidad) es un proceso mediante el cual una forma léxica resulta idéntica a la que se derivó (cfr. Matthews 1979: 131).

5. Cfr. Chavarría (1951), Matluck (1961), Terrel (1975), Hyman (1956), López Morales (1979), Guitart (1981), Cedergren (1973), Lipsky (1966 y 1987$).$

\section{Bibliografia}

Agüero, A. (1962). El español de América y Costa Rica. San José: Lehmann.

Alba, O. (1982). "Estratificación social del español de Santiago: Variación de la /s/ implosiva". Tesis de maestría: Universidad de Puerto Rico. 
Alcina, J. y J.M. Blecua (1975). Gramática española. Barcelona: Arul, 1979.

Alemán (1977). "S final de sílaba implosiva y de final de palabra en el español de Puerto Rico". Tesina: Universidad de Puerto Rico.

Alonso, D. et al (1950). "Vocales andaluzas. Contribución al estudio de la fonología peninsular". Nueva Revista de Filología Hispánica 4:209-30.

Bello, A. (1847). Gramática de la lengua castellana. Buenos Aires: Sopena, 1958.

Carratalá, E. (1980). Morfosintaxis del castellano actual. Barcelona: Labor.

Cedergren (1973). "The interplay of social and linguistic factors in Panama." Tesis doctoral: Universidad de Cornell.

(1978). "En torno a la variación de $s$ final en Panamá: análisis cuantitativo." López M., H. Corrientes actuales de la dialectología del Caribe Hispánico. Río Piedras: Editorial Universitaria, 1978:37-50.

Cepeda, G. (1980). "La formación del plural en español: ni epéntesis ni apócope". Estudios Filológicos 15:51-66.

Chavarría, O. (1951). "The phonemes of Costa Rican Spanish." Language 27, 248-53.

Clegg, H. (1967). "Un análisis acústico de las vocales cubanas". Tesis doctoral: Universidad de Texas.

De la Peña, R. (1985). Gramática teórica y práctica de la lengua castellana. México: Universidad Autónoma de México.

Foley, J. (1967). "Spanish plural formation." Language 46:486-93.

Gagini, c. (1920). Elementos de gramática castellana. San José: Lehmann.

García de D., V. (1951). Gramática bistórica española. Madrid: Gredos.
González, J. (1968). Curso fundamental de gramática castellana. San José [s.n.].

Guitart (1981). "En torno a la sílaba como entidad fonémica en los dialectos del Caribe Hispánico". Boletín del Instituto Caro $y$ Cuervo 36:457-63.

Haden y Matluck (1973). "El habla culta de La Habana: Análisis fonológico preliminar". Anuario de Letras 11:5-33.

Hadlich, R. (1975). Gramática transformativa del español. Madrid: Gredos.

Hammond (1976). "Phonemic restructuring of voiced obstruents in Miami - Cuban Spanish." Aid et al (eds.). Colloquium on Hispanic Linguistics Washington: Georgetown University Press, 1976:42-5.

Harris, J. (1975). Fonología Generativa del español. Barcelona: Planeta.

Henríquez U., P. (1949). Gramática castellana. Buenos Aires: Losada.

Honsa (1965). "The Phonemic Systems of Argentinian Spanish." Hispania 48:275-83.

Hooper, J. (1976). An Introduction to Natural Generative Phonology. New York: Academic Press, Inc.

Hundley (1983). "Linguistic Variation in Peruvian Spanish: Unstressed vowel and /s/." Tesis doctoral: Universidad de Minesota.

Hyman, R. (1956). "[n] as a allophone denoting open juncture in several Spanish American Dialects." Hispania 39:293-9.

Lenz, R. (1925). La oración y sus partes. Madrid: Centro de Estudios Históricos.

Lipski, J. (1986). "Realización de /s/ y /n/ en el dialecto isleño de Luisiana. Remanentes del español canario en Norteamérica". Actas de II Congreso Internacional sobre el español de América. México: Universidad Autónoma de México:321-6. 
(1987). Fonética y fonología del español de Honduras. Tegucigalpa: Editorial Guaymoras, S.A.

Lope Blanch, J. (ed.) (1977). Estudios sobre el español hablado en las principales ciudades de América. México: Universidad Autónoma de México.

López M., H. (1971). Estudios sobre el español de Cuba. Nueva York: Las Americas Publishing Co.

(1983). Estratificactón social del español de San Juan de Puerto Rico. México: Universidad Autónoma de México.

Lorenzo, E. (1971). El español de boy, lengua en ebullición. Madrid: Gredos.

Marín [s.d.]. Gramática castellana.

Martínez de S., J. (1974). Dudas y errores del lenguaje. Madrid: Paraninfo, 1983.

Matluck, J. (1961). "Fonemas finales en el consonantismo puertorriqueño". Nueva Revista de Filología Hispánica 15:332-42.

Matthews, Ph. (1979). Morfología: Introducción a la teoría de la estructura de la palabra. Madrid: Paraninfo.

Navarro, T. (1939). "Desdoblamiento de los fonemas vocálicos en el andaluz". Revista de Filología Hispánica 1:165-7.

Pérez R., A. (1954). Gramática de la lengua española. Madrid: Tecnos.

Perissinotto, G. (1972). "Distribución demográfica de la asibilación de vibrantes en habla de la Ciudad de México". Nueva Revista de Filologia Hispánica 21:73-9.

Poplack (1980). "Deletion and desambiguation in Puerto Rican Spanish". Language 56:37185.

Quesada, N. (1929). Lecciones de gramática castellana. San José: Lehmann.
Real Academia Española (1920). Gramática de la lengua castellana Madrid: Perlado, Páez y Compañía.

(1973). Esbozo de una nueva gramática de la lengua española Madrid: Espasa Calpe, 1979.

Saltarelli, M. (1970). "Spanish plural formation; Apocope or epenthesis?". Language 46:8696.

Sánchez C., V. M. (1988) "El español en Costa Rica: Pieses, cafeses, cubases" Semanario Universidad. 30 de setiembre de 1988, No. 842:6.

Saporta, S. (1965) "Ordered rules, dialect differences and historical processes." Language: 41:218-24.

Stockwell, R. P. et al (1965). The gramatical structure of English and Spanish. Chicago: University of Chicago Press.

Terrel, T. (1975). "La nasal implosiva y final en el español de Cuba". Anuario de Letras 13:257-71.

(1977a). "Constraints on the Aspiration and Deletion of Final /s/ in Cuban and Puerto Rican Spanish". Bilingual Review 4:35-51.

(1977b). "La aspiración y elisión en el español cubano: implicaciones para una teoría fonológica dialectal". Lope Blanch (ed.).

Vázquez, W. (1953). "El fonema s en el español de Uruguay". Revista de la Facultad de Humanidades y Ciencias. 10:87-94.

Wolf, C. y E. Jiménez (1975). "El yeísmo porteño". Lope Blanch (ed.) (1977).

Zamora E., H. (1941). Gramática castellana. San José: Soley y Valverde. 


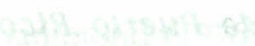

somatis a 